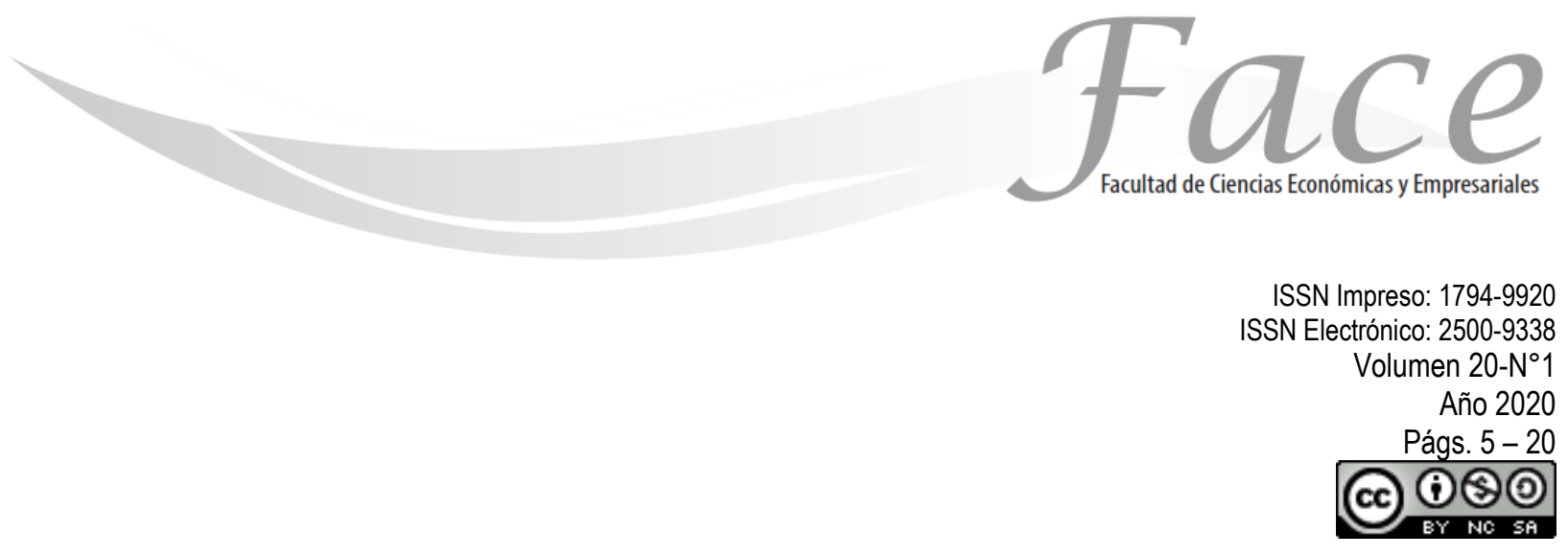

\title{
LA CONTABILIDAD AMBIENTAL Y SU PRODUCTIVIDAD CIENTÍFICA A TRAVÉS DE LA BIBLIOMETRÍA
}

\author{
Alain Castro Alfaro * \\ ORCID: https://orcid.org/0000-0003-1727-7770.
}

Fecha de Recepción: Marzo 30 de 2020

Fecha de Aprobación: Junio 27 de 2020

\section{Resumen:}

La contabilidad ambiental propone información exacta y comprensible a los responsables de las empresas para una mejor toma de decisiones sobre los aspectos financieros y del medio ambiente. Por su parte, la bibliómetrica permite estudiar la producción y la productividad científica y técnica, su influencia y relevancia. El presente artículo tiene como fin el estudio bibliométrico y su relación con la contabilidad ambiental. El tipo de investigación que se utilizó fue el descriptivo cuantitativo, esto porque se caracterizaron indicadores bibliométricos más usuales en este tipo de proyectos. Las bases de datos tomadas en cuenta en el estudio fueron ProQuest, Scielo, Redib y Dialnet y que han publicado sobre la temática entre los años 2016-2017. Se concluye que no son muchas las publicaciones referencias al tema en las bases acotadas.

Palabras clave: Análisis biliométrico, contabilidad ambiental, base de datos, producción científica, estadística.

\footnotetext{
* Magister Gestión de Alta Dirección. (Universidad Federico Villarreal, Perú). Sociólogo. (Universidad Inca Garcilaso de la Vega, Perú). Docente e investigador de la Universidad Rafael Núñez, Cartagena. Colombia. Contacto: alain.castro@curnvirtual.edu.co.
} 


\title{
ENVIRONMENTAL ACCOUNTING AND ITS SCIENTIFIC PRODUCTIVITY THROUGH BIBLIOMETRY
}

\begin{abstract}
:
Environmental accounting provides accurate and understandable information to company managers for better decisionmaking on financial and environmental aspects. For its part, the Bibliometric allows studying scientific and technical production and productivity, its influence and relevance. The purpose of this article is the bibliometric study and its relationship with environmental accounting. The type of research that was used was the quantitative descriptive, this because the most usual bibliometric indicators in this type of projects were characterized. The databases taken into account in the study were ProQuest, Scielo, Redib and Dialnet and they have published on the subject between the years 2016-2017. It is concluded that there are not many publications references to the subject in the limited bases.
\end{abstract}

Keywords: Bibliometric analysis, environmental accounting, database, scientific production, statistic.

\section{CONTABILIDADE AMBIENTAL E SUA PRODUTIVIDADE CIENTÍFICA ATRAVÉS DA BIBLIOMETRIA}

\author{
Resumo:
}

A contabilidade ambiental fornece informações precisas e compreensiveis aos gerentes da empresa para uma melhor tomada de decisão em aspectos financeiros e ambientais. Por sua vez, a Bibliometria permite estudar a produção e a produtividade científica e técnica, sua influência e relevância. $O$ objetivo deste artigo é o estudo bibliométrico e sua relação com a contabilidade ambiental. $O$ tipo de pesquisa utilizada foi a descritiva quantitativa, devido à caracterização dos indicadores bibliométricos mais usuais nesse tipo de projeto. As bases de dados consideradas no estudo foram ProQuest, Scielo, Redib e Dialnet e foram publicadas sobre o assunto entre os anos de 2016-2017. Conclui-se que não há muitas referências bibliográficas ao assunto nas bases limitadas.

Palavras-chave: Análise bibliométrica, contabilidade ambiental, banco de dados, produção científica, estatística. 


\section{INTRODUCCIÓN:}

En el mundo global caracterizado por el cambio acelerado y profundo, aunado a los niveles de crecimiento de las naciones y sus relaciones comerciales (Rivero y Vivas, 2016), las organizaciones, empresas y también los países actúan en ambientes cambiantes, de inestabilidad permanente e incertidumbre. En este contexto turbulento no son suficientes los análisis ambientales, convencionales para el pronóstico de los cambios, que se alimentan desde y con datos exclusivos del pasado, por lo que se hace indispensable que la administración estratégica disponga de nuevas herramientas que expliquen las posibles evoluciones futuras de problemáticas complejas, posibiliten reducir la incertidumbre y sus riesgos asociados permitan adoptar las estrategias más adecuadas para mejorar la toma de decisiones enfocada al futuro (Espindola, 2002).

Es claro que no todas se someten a los cambios y mucho menos se acogen a los nuevos procesos que la ciencia y la tecnología desarrollan día a día con el fin de incrementar valor y coadyudar en el avance, y sostenibilidad de las actividades económicas. Por tanto, la contabilidad como parte de la administración estratégica de cada organización, es una disciplina con visión global, sistémica, dinámica y abierta que explica los posibles futuros no sólo por los datos del pasado, sino fundamentalmente teniendo en cuenta las evoluciones futuras de las variables claves, así como del comportamiento de los actores implicados, de manera que reduzca la incertidumbre, ilumine la acción presente, y aporte con elementos que conduzcan al futuro conveniente o deseado (Villarreal, 2003).

Todo esto, indica que la organización como un sistema abierto establece intercambios con su ambiente para determinar su equilibrio, capacidad de producción y su continuidad. De allí, que las empresas requieren evaluar continuamente las relaciones con el entorno y los componentes, que faciliten su presencia y el alcance de los objetivos permanentemente, por lo que es importante la verificación de información y sus recursos como forma de argumento para los clientes, la orientación de las funciones y la definición de estrategias organizacionales (Mascareñas, 2000).
De manera que, es incuestionable que bajo el sistema económico actual no se logre circunscribir al medio ambiente natural y sus recursos renovables y no renovables dentro cada estructura del mercado, ya que su beneficio simboliza ante los actores económicos un costo, con riesgo de obtener lucros económicos al ser conscientes de sus sustentabilidad, que deben ser esgrimidos a largo plazo y la concerniente evaluación económica del medio ambiente.

Dentro de este contexto, la contabilidad ambiental ha surgido con la necesidad, de medir, cuantificar, registrar, interpretar, analizar, plantear soluciones e informar los daños causados al medio ambiente, proporcionando información confiable, oportuna, comprensible, fidedigna, objetiva e íntegra, permitiendo el diseño de maniobras para la corrección de las falencias, ya que debido al juicio que presenta el proceso de globalización de los recursos naturales no tienen un valor de compromiso racional de los recursos. Por ende, la habilidad de la contabilidad ambiental, debe estar vinculada para formar tácticas para la planeación estratégica en cada empresa con el fin de dar medidas efectivas y eficaces a los problemas, generando un manejo adecuado de los recursos naturales (Gómez y Reyes, 2013).

La existencia de un sistema de contabilidad ambiental ordenado le permite a las organizaciones a tomar en consideración los principios generales y criterios de actuación, cuyo propósito es utilizar la información financiera y no financiera para la toma de decisiones, según la esfera económica, ambiental y social que se logra consolidar como sociedad sostenible. Adicionalmente, la contabilidad ambiental proporciona información exacta y comprensible a los responsables para una mejor toma de decisiones sobre los aspectos que afectan la salud financiera y el medio ambiente (Vergara y De la Espriella, 2016).

De igual forma, se puede definir la contabilidad ambiental como la generación, análisis y utilización de información financiera y no financiera destinada a integrar las políticas económica y ambiental de la empresa, para construir una empresa sostenible. A su vez, identifica, recaba y analiza la información de los costes absorbidos internamente por la empresa 
relacionada al medio ambiente, especialmente los costes relacionados con los desperdicios de materias primas y los costes de producción, eliminación y disposición de residuos (Carvajal, 2017).

Por tal razón, la presente investigación tiene como propósito fundamental Analizar, mediante indicadores bibliométricos, la producción científica en contabilidad ambiental en Colombia 2015-2017, ya que la bibliometría constituye un medio para inferir la producción científica en un área determinada, desde la perspectiva de las fuentes más citadas, las instituciones que las editan y los autores más citados entre otros elementos. Por lo tanto, según White y McCain (1989) la bibliometría es el estudio cuantitativo (métodos matemáticos y estadísticos) acerca del uso de los documentos, siendo un método analítico que mediante la aplicación de estadísticas descriptivas, análisis multidimensional y representaciones gráficas permite medir el desarrollo de la ciencia. En Colombia, por ejemplo, el Indice Bibliográfico Nacional Publindex es la encargada de evaluar de manera bibliométrica a las revistas científicas nacionales e internacionales que deseen ser reconocidas por la misma (Cardona, Lamadrid y Brito, 2018).

En este ejercicio investigativo se propone la tarea de reunir e identificar los desarrollos y avances propuestos por la comunidad académica contableambiental, por medio de los textos publicados en las revistas que forman parte de las bases de datos ProQuest, Scielo, Redib y Dialnet correspondientes al periodo 2015-2017. Desde el contexto de la investigación contable en el trabajo de Beltran, J. P. B., \& Feliciano, L. A. G. (2017), se de muestra como se esta desarrollando la investigación contable en Colombia.

\section{MARCO TEORICO:}

\section{La contabilidad ambiental}

El arte de administrar indefectiblemente está ligado a los sistemas de información contable, de costos y financieros, esto, nos permite, evaluar la situación del negocio y realizar los correctivos de manera oportuna y asertiva para la toma de decisiones y predecir y/o proyectar flujos de efectivo futuro. Definitiva las decisiones sin información confiable, relevante, transparente y oportuna serían como sembrar en tierra no fértil y estaría permeada por la incertidumbre y la desconfianza (Consejo Técnico de la Contaduría Pública, 2008).

A su vez, es importante dentro del sistema contable, el tema relacionado a los tributos, los cuales son fuentes de ingresos que un Estado tiene para poder cumplir con las cargas públicas, siendo este ejercido por la promulgación de leyes tributarias, lo cual crea deberes inherentes a la misma (Moncada, 2016).

El nuevo enfoque de la profesión contable, debatido a nivel internacional es la convergencia a NIIF/ NIC o Estándares Internacionales para Reportes Financieros, emitidos por el IASB; demandando un rol reflexivo, analítico y crítico de los fenómenos que originan los eventos contables y de las actitudes de las personas que los originan, de tal forma que le permita participar en las decisiones estratégicas de las organizaciones (Orellana, 2011).

Por otro lado, los problemas ambientales causados en gran medida por las organizaciones en la actualidad, se deben esencialmente al no reconocimiento y valoración de los recursos naturales, dado que desde el ámbito financiero, la contabilidad intenta amparar una eficiente la inversión en términos económicos y utilización de los recursos, donde se logra igualar el valor agregado que demuestra la inversión medioambiental. . Por tanto, se puede relacionar al conjunto contable tradicional de la actualidad bajo el examen contable medioambiental de índole financiera que hace parte del balance general del Estado de Resultados, del presupuesto, de informes de costos, valoración contable y empresarial, entre otros, que incurre la empresa (Ariza, 2007).

De igual forma, comprender la proporción firme de la racionalidad económica y realidad social en la "existencia de intereses que buscan un progreso y un desarrollo económico, mientras que otros buscan un progreso en la persona, en el individuo. De alguna manera estos dos intereses buscan conciliarse" (Ensuncho, 1995, p. 146). Además, la contabilidad ambiental es social debido que se relaciona con el hombre, puesto que cuando se deteriora el ambiente y 
recursos naturales, se perjudica la calidad de vida de los individuos dentro de cada sociedad.

Por lo tanto, dentro de la contabilidad ambiental es preciso incorporar como sistema informativo, un sistema de cuentas para el mejor económico financiero que exige cada organización mediante costos y gastos, bajo el procedimiento informativo mediante los informes a base de indicadores; que le permite evaluar el impacto de la actividad económica de la empresa en la sociedad, a la vez, logra exponer las voluntades de la industria en cuanto a reposición de inventario, mantenimiento, conservación o deterioro de las condiciones socio-ambientales y de los recursos naturales. (Ensuncho, 1995). Las experiencias financieras contables se amplían en un definitivo argumento social que despliega predominio sobre la manera de rastrear los fundamentos que son tratados soslayando la información que llegará a los más interesados (Larrinaga, 1997).

De igual forma, se precisa que "el propósito de la contabilidad ambiental es proveer información para valorar la sostenibilidad de los capitales" (Mantilla, 2006 , p. 150). La indagación sobre contabilidad ambiental debe ser muy circunspecta por el conocimiento colectivo del problema ambiental, ya pues la comunidad internacional está en la obligación de pronunciar pautas para efectuar procedimientos en las diversas organizaciones que prosiguen la responsabilidad social ambiental, debido al compromiso medioambiental que vorazmente viene ganando espacio en la agenda contable y carteras de servicios corporativos a nivel mundial" (Gómez, 2009, p. 63).

Por otro lado, la contabilidad está fundamentada sobre las políticas financieras a partir de los cambios que presentan las organizaciones; ya que es importante erigir y cambiar los sistemas contables actuales y centrarlos hacia la contabilidad ambiental bajo una responsabilidad social empresarial, donde las empresas reconozcan los sucesos ambientales, sociales y económicos, bajo un medio sostenible (Grisanti, 2015). Se objeta que la contabilidad medioambiental se ampara en los diversos informes contables, con soportado con medidas económicas basadas en falencias ambientales, la cual tiene como propósito luchar por la eficacia en la gestión administrativa empresarial consolidada por los cambios tecnológicos (Gómez, 2009, p. 59).

\section{Valoración Empresarial de la Contabilidad Ambiental}

La influencia de la economía dentro de la empresa ha puesto de manifiesto que el éxito empresarial se basa en la búsqueda de las fuentes de información más objetivas posibles acerca del negocio, a fin de comparar el valor que la empresa y poder tener un precio de mercado entre las partes. Al respecto, López (2000), la valoración de empresas fija el valor de una unidad económica en un momento determinado, a su vez, toma como base supuestos que puedan afectar la valoración final, tales como: oportunidades del mercado, situación política, situación económica del país o de una región determinada, entre otros.

Por su parte, Caballer (1998), señala que la valoración de empresas determina el valor o valores de una empresa con el fin de determinar los procesos en base a las informaciones de carácter técnico y económico. De igual forma, López y De Luna (2001), expresan que valorar se establece en supuestos correctos, emitiendo una opinión y un juicio de valor de la realidad económica empresarial.

En este orden de ideas, Rojo y García (2006) afirman que la finalidad en la valoración se produce cuando el fin de la valoración y los basamentos utilizados no resultan razonables, puesto que para valorar una realidad económica es importante conocer la realidad empresarial, es decir, hay que saber del negocio. Por consiguiente, Martínez (2012), afirma que valorar una empresa "es importante considerar el modelo de valoración que se está aplicando, entendiendo los problemas que se trata de valorar, los supuestos teóricos y prácticos que se emplearán" (p. 20). De aquí que, en el mundo empresarial la valoración define cuantitativamente la realidad existente de la organización, y a su vez, demuestra las conclusiones operativas.

Seguidamente, Blasco y Moya (2012) sostiene que la valoración permite identificar los elementos subjetivos empleados, con temas concretos y las realidades económicas bajo diversos puntos de vista. Por tal razón, toda valoración se contrasta con los resultados, fundamentándose en la capacidad de llevar a la práctica el escenario elegido.

Por otra parte, hablando de mercado de capitales, estos están formadas por un mercado bancario y uno no 
bancario, siendo este último más conocido como mercado de valores (Arrieta, Lora y Sánchez, 2018).

\section{Competitividad}

Para Porter (2000), la competitividad es un proceso que genera $y$ fortalece las capacidades organizacionales, el cual va más allá de la productividad, enfrentando cambios del entorno de manera exitosa, y transformando las ventajas comparativas en sustentabilidad para alcanzar niveles de desarrollo elevados de la empresa. De igual forma, sostiene que la competitividad en las empresas logra emplear sus factores para la producción de sus productos y ofrecer un servicio valioso como proceso de una efectiva productividad, para ello, la organización debe diseñar estrategias donde se pueda motivar, capacitar y orientar los recursos con el fin de lograr los objetivos trazados de manera exitosa (García, Paz y Cantillo, 2019). Igualmente, tener claro que el desempeño organizacional y el bienestar guardan una estrecha vinculación, esto porque mientras más los individuos se siente bien en lugar de trabajo mayor podrá ser su rendimiento (Castro, 2018).

Por otra parte, cuando se habla de competitividad no se puede obviar a la responsabilidad social corporativa, donde las organizaciones de manera voluntaria colaboran en determinados ámbitos del desarrollo social, ambiental y comunal de su zona de influencia (Blanco, 2016), esto traerá una mejor percepción hacia la empresa lo cual puede repercutir en su marca, teniendo presente que, desde el punto de su público interno, el capital humano es un factor de innovación en las organizaciones (Alzamora, 2018). A su vez, se debe tener presente que la responsabilidad social es para las empresas conocer el impacto que generan sus operaciones en los distintos ámbitos donde se relaciona, teniendo en cuenta que la perspectiva de los actores internos y externos en pos de la mejora de la gestión de manera eficiente (Díaz y Bernal, 2017; Salas, 2018).

Por su parte, Villarreal (2006), plantea que la competitividad representa un proceso centrado que genera las capacidades productivas y organizacionales que, desafiando cambios del entorno, de manera exitosa puede lograr condiciones indispensables para alcanzar niveles de desarrollo elevados. Ahora, para que ese logro se pueda dar, se debe de promover una buena planeación estratégica, de tal forma que la empresa pueda enfrentarse a los retos de su entorno y conseguir sus objetivos misionales (Bernal, 2018), teniendo presente que la competitividad financiera es aquel resultado como producto de los cursos de acción competitivos que fueron tomados en cuenta por la organización de tal forma que se tenga una visión clara de ella (Soto, 2016). Sin embargo, lo anterior no podría tener buenos resultados sino se cuenta con una buena información financiera debido a que su carencia no se podrán formular conclusiones sobre la realidad de la empresa (Alzamora, 2016).

De este planteamiento se deduce que la competitividad, según Hernández (2000), es el estadio por el cual deben transitar las empresas para obtener su camino hacia el éxito, ofertando productos $\mathrm{u} / \mathrm{o}$ servicios de alta calidad, con precios atractivos y plazos de entrega oportunos y confiables, logrando de esta forma ventajas competitivas de su producción en otros mercados.

Por tal razón, la competitividad consigue satisfacer las necesidades del entorno, por lo que las empresas están presta tener capacidad de adaptarse al ambiente externo con un intercambio permanente con él, desarrollando su capacidad en cuanto a los recursos que dispone para sostenimiento en los mercados y su crecimiento a mediano y largo plazo.

\section{Finanzas corporativas}

Las finanzas corporativas para Koller, Goodhead y Wessels (2005), crean valor mediante el uso eficaz de los recursos financieros que se encuentran en el estudio de los activos tanto tangibles como intangibles y en los que la empresa deba invertir. Asimismo, las finanzas corporativas toman las decisiones de financiación, mediante la obtención de fondos de la empresa y que pueda adquirir los activos en cuanto a las decisiones operativas y financieras.

De manera que, las finanzas corporativas enfatizan el análisis para conocer qué recursos financieros cuenta la empresa y la forma de hacer más inversiones dentro de ella para generar más utilidades. Además, las finanzas contribuyen en el buen desarrollo de la empresa, obtenido como oportunidad inyectar capital y aumentar las acciones. A continuación se describen las diferentes partes de las finanzas corporativas: 
- Las decisiones de inversión, se centran en los activos reales (tangibles o intangibles) en los que la empresa debería invertir.

- Las decisiones de financiación, se obtienen a través de los fondos que provienen de los inversores y los activos financieros emitidos por la empresa).

- Decisión de dividendos, se logran para tomar decisiones concretas y la obtención de un buen desarrollo empresarial.

- Las decisiones directivas, decisiones operativas y financieras entre los socios que son fijados para logro de los objetivos y metas deseadas.

De lo anterior, se afirma que para cualquier tipo de empresa es importante las finanzas debido a que la inversión de todo capital genera utilidades para el crecimiento de la organización, por ello es importante tomar en consideración las amenazas dentro de la empresa, fijando objetivos para enfrentar los problemas y el logro de mantener las herramientas necesaria que sirvan de solución y tomar las decisiones adecuadas para el buen funcionamiento de la empresa. Sin embargo, los riesgos financieros inciden en la rentabilidad, en las entradas y en el nivel de inversión (Albarracín, García y García, 2017). También se destaca la generación de competitividad que se puede dar en la gestión financiera desde una perspectiva en la finanzas corporativas planteadas por Berrio, Pérez, y Brito (2017).

\section{Auditoría Ambiental}

La auditoría ambiental se define como investigación metódico, versado, constante e imparcial, por las diversas entidades reglamentadas, de operaciones y prácticas de todas las organizaciones relacionadas para el acatamiento de obligaciones ambientales, y que cuyas acciones logren producir impacto ambiental y queden sujetas a control gubernamental (EPA, 2013). Por tanto, la coexistencia en que la Auditoría Ambiental permite su valoración según el sistema de gestión, control y evaluación ambiental de cada empresa, ya que es el instrumento que permite comprobar si el sistema funciona apropiadamente.

\section{Contabilidad Ambiental y las Normas Internacionales de Información Financiera}

En Colombia, tiene un impacto positivo al adaptarse al proceso de las normas internacionales los cuales llegan no afectar los resultados y patrimonios de las empresas, es importante que estén dispuestos a ajustarse al proceso de adaptación y entiendan la importancia y el alcance de un cambio fundamental en la forma en que las empresas medirán los resultados y se presentaran al mundo exterior ya que es un proceso paulatino el cual tardará varios años en implantarse por completo, pero esto no conllevaría a consecuencias en el departamento de contabilidad (García y otros, 2012).

Con la aprobación del reglamento que establece la aplicación de las normas internacionales de contabilidad, la unión europea se convierte en el principal motor para la expansión de unas normas de alta calidad concebidas desde una perspectiva mundial, redundando en beneficio de una información financiera transparente y comparable más allá de sus fronteras. (Espinosa-Flórez, 2002).

Además, la contabilidad, como ciencia y técnica de la información financiera y operacional de los entes económicos, debe cumplir con exigencias cada día mayor, por ello, las crecientes necesidades de información para planificar, financiar y controlar el desarrollo nacional requeridas por un sinnúmero de entidades del estado (Barrientos y Sarmiento, 2009).

Siguiendo con el mismo autor, la contabilidad requiere para las necesidades de información de trabajadores e inversionistas; el desarrollo de sofisticados procedimientos administrativo-contables para controlar los enormes volúmenes y complejas operaciones de las empresas, que han hecho imprescindible la herramienta de la computación y las complicaciones que involucra utilizar la moneda como patrón de medida, en países afectados por inflación, plantean un verdadero desafío a nuestra profesión.

Las normas han sido producto de grandes estudios y esfuerzos de diferentes entidades educativas, financieras y profesionales del área contable a nivel mundial, para estandarizar la información financiera presentada en los estados financieros. Las NIC, como se le conoce popularmente, son un conjunto de normas o leyes que establecen la información que deben presentarse en los estados financieros y la forma en que esa información debe aparecer, en dichos estados (Amat, 2003).

De manera que, las NIC no son leyes físicas o naturales que esperaban su descubrimiento, sino más bien normas que el hombre, de acuerdo sus 
experiencias comerciales, ha considerado de importancias en la presentación de la información financiera. Asimismo, son normas de alta calidad, orientadas al inversor, cuyo objetivo es reflejar la esencia económica de las operaciones del negocio y presentar una imagen fiel de la situación financiera de una empresa (Valencia y otros, 2007).

Ante cualquier fenómeno armonizador en el ámbito internacional, lo primero que hay que delimitar con claridad es el modelo objeto de aceptación con carácter general. Al respecto, la International Organization of SecuritiesCommission (IOSCO), encargó en 1995 al International Acounting Standards Committee, en la actualidad International AccountingStandardsBoards (IASB), la tarea de elaborar un cuerpo básico de normas contables que pudieran ser aceptadas en los mercados financieros internacionales y por tanto, aplicadas por las empresas que desearan que sus valores cotizaran en los citados mercados (Cardona y Zapata, 2004).

Tras una profunda reforma organizativa en 2001 el IASB se ha convertido en un organismo independiente, regido por una Fundación que cuenta con los siguientes órganos a través de los cuales desarrolla sus funciones:

Los administradores, que nombran a los miembros de los diferentes consejos y comités dentro del IASB captan fondos, aprueban el presupuesto y tienen responsabilidad para acometer cambios en la constitución del órgano.

El consejo es el órgano técnico más importante dentro del IASB; está compuesto por catorce miembros con experiencia y conocimientos técnicos, la mayoría de los cuales ejerce su misión tiempo completo.

El consejo asesor de normas, compuesto por unos treinta miembros, que es el vehículo formal para que personas de diferente procedencia geográfica y profesional puedan asesorar al consejo sobre las prioridades de actuación en la normativa contable y las posibles implicaciones de las normas.

El comité de interpretaciones, compuesto por doce miembros que tienen la misión de estudiar los problemas que surjan al aplicar las normas con el fin de realizar la exégesis de las mismas en situaciones concretas.
Los grupos de trabajo, constituidos por diferentes expertos interesados en la elaboración de documentos de discusión y proyectos de normas, siguiendo la agenda previamente acordada por el consejo, que es, en todo caso el órgano encargado de la aprobación de todos los documentos y pronunciamientos oficiales del IASB (Rojo y Caba, 2003).

Por otro lado, los objetivos del IASB establecidos en su constitución, son los siguientes:

Desarrollar para el interés público, un conjunto de normas generales de contabilidad comprensibles y aplicables, que permitan exigir una alta calidad, transparencia y comparabilidad dentro de la información contenida en los estados financieros y otra información a suministrar, que ayude a los partícipes en los mercados financieros mundiales, así como a otros usuarios, en la toma de decisiones económicas; promover el uso y aplicación de dichas normas. $Y$ conseguir la convergencia entre las normas contables de ámbito nacional y las Normas Internacionales de Contabilidad, en soluciones de elevada calidad técnica (Rojo y Caba, 2003).

\section{La Bibliometría}

En análisis bibliométrico de las investigaciones desarrolladas en los diferentes contextos es un tema que recientemente ha tomado auge debido a la necesidad de agrupar las investigaciones y reconocer de esta manera el avance que se ha dado en investigación de una población. Las definiciones de bibliometría son diversas y se articulan entre los siguientes: conjunto de estudios que tratan de cuantificar el proceso de comunicación escrita y la naturaleza y evolución de las ciencias, mediante el recuento y análisis de las diferentes características de dicha comunicación (Rodríguez y Ramón, 2007).

Por su parte, Camps (2007), plantea otra definición la cual se refiere a la ciencia que permite el análisis cuantitativo de la producción científica a través de la literatura, estudiando la naturaleza y el curso de una disciplina científica: el uso de indicadores bibliométricos para analizar la actividad investigadora de un equipo científico, un área o un país, se bases en que las publicaciones científicas son un resultados esencial de dicha actividad. Un nuevo conocimiento adquiere valor cuando se da a conocer y se difunde, ya que es así como progresa la ciencia; además de esto, para Duque, Cervera y Rodríguez, 2006), la 
bibiometría permite valorar la calidad científica e influencia tanto del trabajo como de las fuentes, se define como la aplicación de métodos matemáticos y estadísticos a libros y otros medios de comunicación escrita.

Para Aguilar, López, Barreto, Bolena, Rodríguez y Vargas (2007), la bibliometría es un método que usa recursos cuantitativos y analíticos que mediante la aplicación de estadísticas descriptivas, análisis multidimensional y representaciones gráficas permite medir el desarrollo de la ciencia. Este método es de gran utilidad por cuanto permite con precisión orientar la formulación de políticas, planes y estrategias para orientar las dinámicas de desarrollo de la producción de conocimiento de una comunidad. A su vez, el mismo autor expresa que la bibliometría hace parte de la ciencia métrica y está ligada a la bibliografía y a las fuentes de información, esta relación es bastante importante ya que los estudios de esta índole se llevan a cabo a través de los análisis de estas fuentes y constituyen el sustento sobre el cual se mide o clasifica la producción científica. Es aquí donde se da el inicio de este proceso, ya que los estudios bibliométricos se llevan a cabo a partir de los análisis de las fuentes de información e, inclusive, los mismos investigadores de esta disciplina hacen alusión a las fuentes de información como el medio sobre el cual se valen las técnicas bibliométricas, que al mismo tiempo, están relacionadas con los métodos estadísticos.

Por su parte, Agudelo (2003), expone que la ciencia bibliométrica es de gran utilidad e interés puesto que permite la búsqueda más especializada y organizada en una temática específica, el cual es orientar al autor hacia la obtención de mejores opciones para publicar sus trabajos en función de las áreas desarrolladas en diferentes publicaciones, crea una relación de la comunidad científica y un acercamiento a otros grupos de trabajo de temáticas afines.

\section{METODOLOGÍA:}

La presente investigación fue de tipo descriptiva, debido a que se llevó a cabo una revisión bibliográfica para obtener las inferencias necesarias con el propósito de alcanzar la solución de los objetivos.

El presente proyecto fue de carácter cuantitativo de tipo descriptivo, dado que se basa en un análisis de la producción científica para realizar un análisis bibliométrico basada en la producción científica en contabilidad ambiental en Colombia 2015-2017 (Sabino, 2002). Para el análisis de la información se tomó en consideración la biometría, cuyas vertientes se focalizan en la producción científica dado el tratamiento y análisis cuantitativo de las publicaciones científicas (De Filippo, et al., 2011). De igual forma, dentro del estudio se aplican los indicadores bibliométricos para determinar la productividad de los autores, variación cronológica de trabajos publicados, número de trabajos publicados y número de autores por trabajos.

Se utilizaron fuentes secundarias, tales como ensayos, artículos científicos, libros y otros capaces de brindar fundamentación teórica al proyecto.

El instrumento que se tomó en consideración fueron los indicadores bibliométricos como las características bibliométricas que describe el año de publicación, base de datos, país donde se efectuó el estudio, número de páginas y número de referencias. Se logró identificar y analizar 42 artículos científicos que guardan relación con la producción científica en contabilidad ambiental en Colombia, publicados en la base de ProQuest, Scielo, Redib y Dialnet correspondientes al periodo 2015-2017.

Una vez recabada la información, se logró determinar los descriptores identificando los indicadores bibliométricos para la sistematización de la información. Posteriormente, se procedió a la búsqueda de los datos mediante la base de datos ProQuest, Scielo, Redib y Dialnet para el período 2015-2017, donde se logró la tabulación respectiva utilizando como herramienta el programa Excel y luego se procedió a analizar la información tomando en consideración las características bibliométricas. 


\section{RESULTADOS:}

Para el siguiente análisis se tomó en cuenta las características bibliométricas y metodológicas de 42 artículos sobre la producción científica en contabilidad ambiental en Colombia 2015-2017.

\section{Identificación de las revistas y los autores que han publicado artículos sobre la contabilidad ambiental en Colombia por medio de las bases de datos ProQuest, Scielo, Redib y Dialnet correspondientes al periodo 2015-2017}

La Tabla 1 muestra que el 10\% de los artículos están publicados en la Revista Contaduría Universitaria de Amtioquia, Criterio Libre y Revista Ambiente Contábil, el $7,5 \%$ en Revista Publicando, el $5 \%$ en Revista Contabilidad y Negocios, Revista Ciencias Holguín, Visión Gerencial y Saber, Ciencia y Libertad, respectivamente, y el 2,5\% del resto de los artículos poseen menor rango de publicaciones.

Tabla 1. Nombre de la Revista

\begin{tabular}{|c|c|c|}
\hline Nombre de la fuente & Frecuencia & Porcentaje \\
\hline Contaduría Universidad de Antioquia & 4 & 10 \\
\hline Criterio Libre & 3 & 7,5 \\
\hline Contabilidad y Negocios & 2 & 5 \\
\hline Cuadernos de Contabilidad & 1 & 2,5 \\
\hline Ambiente y Desarrollo & 1 & 2,5 \\
\hline Revista Ambiente Contábil & 4 & 10 \\
\hline Ciencias Holguín & 2 & 5 \\
\hline Revista DYNA & 1 & 2,5 \\
\hline Revista Infraestructura Vial & 1 & 2,5 \\
\hline Ágora de heterodoxias & 1 & 2,5 \\
\hline Teuken Bidika & 1 & 2,5 \\
\hline Visión Gerencial & 2 & 5 \\
\hline Monteverdia & 1 & 2,5 \\
\hline Revista Entorno & 1 & 2,5 \\
\hline Quipukamayoc & 1 & 2,5 \\
\hline Bosque Latitud Cero & 1 & 2,5 \\
\hline Revista ENIAC Pesquisa & 1 & 2,5 \\
\hline Revista Visión Contable & 1 & 2,5 \\
\hline AGLALA & 1 & 2,5 \\
\hline El Perito Sustentable & 1 & 2,5 \\
\hline Revista Publicando & 3 & 7,5 \\
\hline REICE & 1 & 2,5 \\
\hline Saber, Ciencia y Libertad & 2 & 5 \\
\hline Panorama Económico & 1 & 2,5 \\
\hline Producción + Limpia & 1 & 2,5 \\
\hline Contaduría y Administración & 1 & 2,5 \\
\hline
\end{tabular}

Fuente: Elaboración propia 
La Tabla 2 demuestra que el 87,5\% delos autores que publicaron pertenecen a la categoría de Aspirantes, el 97,5\% corresponde a la afiliación de los diversos autores correspondientes a las instituciones universitarias y el 82,5\% fueron escritos entre 1 a 3 autores y el $8 \%$. En cuanto a los productores moderados se obtuvo que $12,5 \%$ son autores que mantienen sus publicaciones en la Universidad de Amtioquia, Universidad Pontificia Bolivariana de Colombia, Universidad de Los Andes Venezuela, entre otras.

Tabla 2. Información de los autores

\begin{tabular}{llcc}
\multicolumn{1}{c}{ Indicador } & \multicolumn{1}{c}{ Categoría } & Frecuencia & Porcentaje \\
\hline Autores & Transeúntes Aspirantes & 35 & 87,5 \\
& & & 12,5 \\
Afiliación de los autores & Productores moderados & 5 & 97,5 \\
& Universidades & 39 & 2,5 \\
& Organismos gubernamentales & 1 & 17,5 \\
Número de firmas & Otro $\quad$ refiere & & 82,5 \\
& No a 6 autores & 33 & \\
\hline
\end{tabular}

Fuente: Elaboración propia

Descripción a través de indicadores bibliométricos de los artículos publicados en relación a la contabilidad ambiental en Colombia por medio de las bases de datos ProQuest, Scielo, Redib y Dialnet correspondientes al periodo 2015-2017

Se puede evidenciar en la Tabla 3 la existencia de un número mayor de artículos publicados en el año 2017 con el 47,5\%, por otro lado el $24 \%$ corresponde a los artículos científicos pertenecientes a la base de datos ProQuest. Asimismo, el $40 \%$ de las producciones científicas se elaboraron en Colombia, el 50\% de los artículos tienen un número de páginas entre 12 a 23 , seguidamente el $35 \%$ de los artículos poseen referencias de Más de 40.

Tabla 3. Indicadores Bibliométricos

\begin{tabular}{|c|c|c|c|}
\hline Indicador & Categoría & Frecuencia & Porcentaje \\
\hline \multirow{3}{*}{ Año de publicación } & 2015 & 11 & 25 \\
\hline & 2016 & 10 & 27,5 \\
\hline & 2017 & 19 & 47,5 \\
\hline \multirow{4}{*}{ Base de Datos } & ProQuest & 12 & 24 \\
\hline & Science & 6 & 22 \\
\hline & Redib & 14 & 36 \\
\hline & Dianet & 8 & 18 \\
\hline \multirow{10}{*}{$\begin{array}{l}\text { País donde se realizó el } \\
\text { estudio }\end{array}$} & Brasil & 5 & 12,5 \\
\hline & Colombia & 16 & 40 \\
\hline & Costa Rica & 1 & 2,5 \\
\hline & Cuba & 4 & 10 \\
\hline & Ecuador & 4 & 10 \\
\hline & El Salvador & 1 & 2,5 \\
\hline & México & 2 & 5 \\
\hline & Nicaragua & 1 & 2,5 \\
\hline & Perú & 3 & 7,5 \\
\hline & Venezuela & 3 & 7,5 \\
\hline \multirow{4}{*}{ Número de páginas } & 6 a 12 & 8 & 20 \\
\hline & 12 a 23 & 20 & 50 \\
\hline & 24 a 40 & 12 & 30 \\
\hline & Más de 40 & 0 & 0 \\
\hline \multirow{4}{*}{ Número de referencias } & 6 a 10 & 7 & 17,5 \\
\hline & 12 a 22 & 8 & 20 \\
\hline & 24 a 38 & 11 & 27,5 \\
\hline & Más de 40 & 14 & 35 \\
\hline
\end{tabular}

Fuente: Elaboración propia 


\section{CONCLUSIONES:}

En el presente estudio se ha utilizado el análisis bibliométricos en cuanto a la producción científica en contabilidad ambiental en Colombia, con el fin de describir el nivel de divulgación de información ambiental en las revistas contables colombianas divulgadas en la base de datos ProQuest, Scielo, Redib y Dialnet. El procedimiento se aplicó a las revistas de laContaduría Universidad de Antioquia, Criterio Libre, Contabilidad y Negocios, Cuadernos de Contabilidad, Ambiente y Desarrollo, Revista Ambiente Contábil, Ciencias Holguín, Revista DYNA, Revista Infraestructura Vial, Ágora de heterodoxias, Teuken Bidika, Visión Gerencial, Monteverdia, Revista Entorno, Quipukamayoc, Bosque Latitud Cero, Revista ENIAC Pesquisa, Revista Visión Contable, AGLALA, El Perito Sustentable, Revista Publicando, REICE, Saber, Ciencia y Libertad, Panorama Económico, Producción + Limpia y Contaduría y Administración. Por tanto, es importante resaltar que, con el presente ejercicio investigativo, se han querido ofrecer unos pertinentes para la perspicacia del fenómeno experimentado, a fin de igualar, dentro de la entidad académica que desenvuelve el tema medioambiental en la contabilidad, los posibles espacios de exploración académica (Rodríguez, y Valdés, 2018).

Resulta claro, que la divulgación académica de del tema contabilidad ambiental resulta pertinente para Bernal y Santos (2018) afirmando que mediante este tipo de investigaciones se logra dinamizar la conexión de contabilidad financiera y ambiental para detener los efectos de las acciones medioambientales de las diversas organizaciones empresariales en sus estados financieros, ya que la opinión de diversos autores de las Revistas Internacionales demuestran los logros alcanzados en cuanto a la contabilidad ambiental se refiere. Por ello, los diferentes informes que describen la contabilidad ambiental suministran a los diferentes usuarios herramienta esencial que fungen para la toma de decisiones con base a una información integral emitida por esta rama de la contabilidad, además se espera que dicha información abarque los ámbitos del desarrollo sostenible: económico, ambiental y social.
De igual forma, la publicación científica expuesta por Linares y Suárez (2017) demuestran el avance de las publicaciones científicas en el campo de la contabilidad académica, ya que cada revisión teórica de la economía ambiental y la economía ecológica en todos sus ámbitos enfatizan el grado de los costos ambientales que constituyen un punto importancia en la retórica internacional. Además, se observa en la literatura un favorecimiento hacia la innovación ecoeficiente que repercute en los costos para cada organización, propiciando herramientas de costeo ambiental que reportan valores monetarios y que aprovechan la plataforma estratégica de la industria que responden a las necesidades ambientales actuales.

El caso de la contabilidad ambiental para los científicos académicos no se limita a la maximización de utilidades para las organizaciones como las expectativas de las gobiernos y científicas indican que se entiende los costos medioambientales desde una perspectiva más amplia comprendiendo lo siguiente: (a) obligaciones económicas al pagar los impuestos y cánones mineros respectivos; (b) dimensión legal, al cumplir con la legislación desde la elaboración de un estudio de impacto ambiental y (c) enfoque filantrópico, al llegar a acuerdos que incluyen la construcción de infraestructura para favorecer el desarrollo de las comunidades (Quintero y Ramírez, 2016).

En futuras investigaciones, será importante abordar el cambio que ha tenido el humanismo en organizaciones que ahora funcionan como sistemas abiertos, con contextos de trabajo tan diferentes como el home office y la economía colaborativa. 


\section{CONCLUSIONES:}

La exigencia relacionada con la información financiera y económica es cada vez más dilatada y necesariamente tiene que estar vinculada con las premisas y exigencias que manejan las organizaciones en la actualidad, la cual requiere trasparencia que al mismo tiempo se manifiesta en una serie de propiedades y características como pueden ser la objetividad en los informes, generalidad, utilidad de la misma, así como su homogeneidad. Por otra parte, se encuentra el hecho de que debe existir una gran responsabilidad al asumir con gran compromiso la información corporativa, producto de una sucesión que incluye no solamente al Consejo Financiero de la empresa sino también gestores comerciales, analistas de finanzas, personal contable, auditores, entes reguladores, personajes económicos, entre otros.

Es esta pues una oportunidad para que las organizaciones puedan dar a conocer su realidad empresarial, sus oportunidades y realidades con lo cual pueden indagar oportunidades de crecimiento y financiación no solo a nivel nacional, sino más allá de las fronteras, por el hecho de que con las normas establecidas para mantener una efectiva contabilidad ambiental como parte de su estructura financiera son muchos los analistas y revisores de la información contable que pueden entender cuál es la situación de la empresa en cuanto a solvencia, rentabilidad, capacidad de pago, activos y pasivos reales, entre otros.

De igual forma, la adopción de las normas internacionales de información es una oportunidad de crecimiento sustancial, ya que la apertura a otros mercados se genera desde el momento en el que el lenguaje se hace universal y se hace entendible la realidad de la contabilidad ambiental. Los revisores de la información financiera dentro de cada organización podrán hacer las respectivas comparaciones una vez se tenga un lenguaje económico unitario con el que se puedan cotejar las actividades y desarrollos de la empresa en un periodo determinado de tiempo, es entonces esta situación la que permite a las organizaciones con necesidades de financiamiento hacerle frente a la situación medioambiental y demostrar sus finanzas, en virtud de que el analista financiero entenderá dicha información desde cualquier perspectiva.

Cada producción científica registrada en revistas indexadas en ProQuest, Scielo, Redib y Dialnet y en revistas de contables nacionales en Colombia demuestra su conocimiento teórico de la contabilidad ambiental, desarrollo de propuestas que satisfagan las demandas del contexto y que consideren otras herramientas que no necesariamente vinculen valores monetarios. Por tanto, es importante que Colombia se integre a la investigación de esta materia a través de estudios teóricos y empíricos que establezcan un horizonte de mejores prácticas para la industria nacional y permitan nuevos desarrollos adaptados al contexto.

Por tal razón, los indicadores bibliométricos de la contabilidad ambiental demuestran que de responsabilidad académica establece la existencia de mediciones ordenadas que toma en cuenta el compromiso por parte de las Universidades y Revistas Internacionales en el compromiso de la producción teórica en cuanto al mejoramiento de la calidad ambiental y la educación y concientización ambiental. Asimismo, se establece el gerenciamiento del impacto ambiental, buscando reducirlo y siendo consciente de que se genera no solamente por las actividades que se desarrollan dentro de la empresa sino también por los usos que las comunidades y personas en general le den al medio ambiente y todo esto afecta al entorno.

\section{REFERENCIAS:}

Aguilar, M., López, W., Barreto, I., Bolena, Z., Rodríguez, C. y Vargas, E. (2007). Análisis bibliométrico de los trabajos de grado del área organizacional de la facultad de psicología de la Universidad Santo Tomás. Diversitas, 3 (2), 317-334. Disponible en https://www.redalyc.org/pdf/679/67930211.pdf

Agudelo, D. (2003). Análisis bibliométrico de las revistas relacionadas con psicología de la salud editadas en castellano. Universidad de Granada, 15 (4), 507-516 
Arrieta Rodríguez, D. A., Lora Guzmán, H., \& Sánchez González, J. (2018). Análisis de las empresas de Cartagena del sector logístico portuario basado en el modelo de configuración estratégica de Danny Miller. Aglala, 9(1), 62-90. Recuperado de http://revistas.curnvirtual.edu.co/index.php/agla la/article/view/1182

Albarracín Muñóz, M., García Arenas, L., \& García, C. (2017). Riesgo financiero: una aproximación cualitativa al interior de las mipymes en Colombia. Aglala, 8(1), 139-160. https://doi.org/https://doi.org/10.22519/221573 $\underline{60.1029}$

Alzamora, E. (2018). El poder del conocimiento y de la información como generador de valor en las organizaciones. Conocimiento global 3(1), 1429. Recuperado a partir de http://conocimientoglobal.org/revista/index.php/ cglobal/article/view/3

Alzamora Taborda, E. (2016). Impacto de las prácticas de auditoría en la disminución de fraude en las organizaciones. Enfoque Disciplinario, 1(1), 3540. Recuperado a partir de http://enfoquedisciplinario.org/revista/index.php lenfoque/article/view/7

Amat, O. (2003). Comprender las Normas Internacionales de Contabilidad NIC. 2da. edición ampliada. Barcelona-España: Ediciones Gestión 2000.

Ariza, E. D. (2007) Luces y sombras en el Poder Constitutivo de la Contabilidad Ambiental. Revista Facultad de Ciencias Económicas, 15(2), 45-60.

Barrientos, E. \& Sarmiento, P. (2009).Normas Internacionales de Información Financiera NIIFIFRS. Ejemplos ejercicios y casos prácticos comentados. Bogotá: Cargraphics.

Bernal, M. y Santos, E., (2018). Una mirada a la contabilidad ambiental. Postulados y retos. Contaduría Universidad de Antioquia, 73, 199209.
Bernal Payares, O. (2018). Planeación estratégica y sostenibilidad corporativa. Conocimiento Global, 3(1), 50-55. Recuperado a partir de http://conocimientoglobal.org/revista/index.php/ cglobal/article/view/27

Berrio, B. J. D. Á., Pérez, H., \& Brito, R. R. V. (2017). Gestión financiera y competitividad de las empresas prestadoras de servicios turísticos en la frontera colombo-Venezolana. FACE: Revista de la Facultad de Ciencias Económicas y Empresariales, 16(2), 38-53. Recuperado de: http://revistas.unipamplona.edu.co/ojs_viceinv es/index.php/FACE/article/view/2289

Beltran, J. P. B., \& Feliciano, L. A. G. (2017). Tendencia de Investigación contable en Colombia: Teoría - Práctica, una mirada desde las revistas contables colombianas 2011 a 2015. FACE: Revista de la Facultad de Ciencias Económicas y Empresariales, 17(2), 68-80. Recuperado de: http://revistas.unipamplona.edu.co/ojs_viceinv es/index.php/FACE/article/view/2228

Blasco, A. y Moya, I. (2012). Apuntes de mercados financieros y valoración de empresas el Máster en Dirección Financiera y Fiscal. Valencia: Universidad Politécnica de Valencia.

Blanco Rangel, I. (2016). Apuntes sobre Colombia a propósito de su internacionalización y otros desafíos. Aglala, 7(1), 209-224. Recuperado de

http://revistas.curnvirtual.edu.co/index.php/agla la/article/view/905

Carvajal, F. (2017). Avances y desafíos de las cuentas económico-ambientales en América Latina y el Caribe. Disponible en: https://repositorio.cepal.org/bitstream/handle/1 1362/43314/1/S1700990_es.pdf

Cardona, J. y Zapata, Á. (2004). Estándares Internacionales para la Formación del Contador Público. En: Revista Contaduría, 44, 152-163.

Cardona Arbelaez, D., Lamadrid Villarrreal, J., \& Brito Carrillo, C. (2018). La gestión y dirección del talento humano desde el análisis sobre clima organizacional y sus dimensiones. Un estudio de caso. Aglala, 9(1), 154-176. Recuperado de 
http://revistas.curnvirtual.edu.co/index.php/agla la/article/view/1185

Castro Alfaro, A. F. (2018). Bienestar como fuente de actuación organizacional. Enfoque Disciplinario, 3(1), 1-18. Recuperado a partir de http://enfoquedisciplinario.org/revista/index.php lenfoque/article/view/10

Camps, D. (2007). Estudio bibliométrico general de colaboración y consumo de la información en artículos originales de la revista Universitas Médica, periodo 2002-2006. Universitas Médica, 48 (4), 358-365. Recuperado de https://www.redalyc.org/pdf/2310/2310186700 $\underline{02 . p d f}$

Consejo Técnico de la Contaduría Pública (2008). Ejercicio profesional de la revisoría fiscal. Disponible en: https://www.aesa.org.co

Caballer, V. (1998). Métodos de Valoración de Empresas. Pirámide. Madrid.

De Filippo, D.; Sanz-Casado, E.; Urbano, C.; Ardanuy, J., y Gómez, I. (2011). El papel de las bases de datos institucionales en el análisis de la actividad científica de las universidades. Revista Española de Documentación Científica, 34(2), 165-189.

Díaz Ortíz, A., \& Bernal Payares, O. (2017). Caracterización del estado de responsabilidad social empresarial en los hoteles cinco estrellas de la ciudad de Cartagena - Caso hotel Holiday Inn Morros Cartagena. Conocimiento Global, 2(1), 56-63. Recuperado a partir de http://conocimientoglobal.org/revista/index.php/ cglobal/article/view/16

Duque, E., Cervera, A. y Rodríguez, C. (2006). Estudio bibliométrico de los modelos de medición del concepto de calidad percibida del servicio en internet. Revista Innovar, 16 (28), 223-243. Recuperado de https://www.javeriana.edu.co/biblos/tesis/cienci as/tesis209.pdf

Espindola, O. (2002). Competencia y competitividad diseñada para organizaciones latinoamericanas. México: Mc Graw Hill.
Espinosa-Flórez, G. (2002). Transparencia y Comparabilidad: premisas fundamentales en los procesos internacionales de regulación contable. Revista Legis del Contador, 65-73.

Ensuncho, J. (1995). Contabilidad social. Medellín, Colombia: Centro Colombiano de Investigaciones Contables.

Enviromental Protection Agency (EPA) (2013). Financial Accounting Standards Board (FASB). Generally Accepted Accounting Principles (GAAP). Disponible en: http://www.epa.gov/espanol/.

Fernández, P. (2004). Valoración de empresas. 3ra. Ed. España: Gestión 2000.

García Guiliany, J., Paz Marcano, A., \& Cantillo Campo, N. (2019). Estrategia y habilidades para la competitividad: caso de pymes del sector construcción en Barranquilla. Aglala, 10(1), 312-339. Disponible en

http://revistas.curnvirtual.edu.co/index.php/agla la/article/view/1349

Gómez, M. (2009). Tensiones, posibilidades y riesgos de la contabilidad medioambiental empresarial. Contaduría Universidad de Antioquia, 54, 5578.

Grisanti, Á. (2015). La Responsabilidad Social Empresarial. Ejemplo práctico de gestión ética en y para los negocios. Visión Contable, 13, 134-157.

Gómez, M., y Reyes Rodríguez, M. (2013). La Contabilidad Medio Ambiental y los impuestos Ambientales aplicados en las PYMES: caso empresas agrícolas del valle de Autlán Jalisco, México. Global Conference On Business \& Finance Proceedings, 8(1), 1123-1134.

Hernández, E. (2000). Competitividad industrial en México. México: Plaza y Valdés Editores.

Koller, T., Goodhead, M. y Wessels, D. (2005): Valuation. Measuring and managing the Value of Companies. John Wiley. Nueva York. 
Larrinaga, C. (1997). Consideraciones en torno a la relación entre la contabilidad y el medio ambiente. Revista Española de Financiación Revista Española de Financiación y Contabilidad, 26(93), 957-991.

Linares, M. y Suárez, Y. (2017). Los costos ambientales: un análisis de la producción ientífica en el periodo 1977-2016 y una revisión de herramientas y teorías subyacentes. Criterio Libre, 15(27), 89-114.

López, F. (2000). Valoración de empresas en la práctica. México: McGraw-Hill.

López, F. y De Luna, W. (2001). Valoración de empresas en la práctica. España: McGraw-Hill Interamericana.

Moncada, L. (2016). Cumplimiento de obligaciones tributarias en el libro de compras en Plumrose CA. Conocimiento Global, 1(1), 14-36. Recuperado a partir de http://conocimientoglobal.org/revista/index.php/ cglobal/article/view/21

Orellana, I. (2011). Articulo las normas internacionales de información financiera (N.I.I.F), impacto en el gobierno corporativo y en los impuestos, revista de la Universidad del Azuay, Cuenca Ecuador, edición № 56.

Quintero, A. y Ramírez, A. (2016). Por caminos contrahegemónicos hacia una contabilidad ecológica. Contaduría Universidad de Antioquia, 68, 287312.

Rivero, F., \& Vivas, F. (2016). Análisis de los mecanismos de promoción de exportación de especies acuícolas: camarones (palaemon serratus) en la República Bolivariana de Venezuela durante el periodo 20052009. Conocimiento Global, 1(1), 55-77. Recuperado a partir de http://conocimientoglobal.org/revista/index.php/ cglobal/article/view/24

Rodríguez, D.F. y Valdés, P.C. (2018). Balance de las publicaciones que abordan el concepto de contabilidad ambiental en revistas contables colombianas Revista Visión Contable, 17, 2679.

Rodríguez, P. y Ramón, A. (2007). Abordaje bibliométrico a los servicios blindados por el profesional de la información. Ciencias de la Información 38 (3), 39-50. Recuperado de https://www.redalyc.org/pdf/1814/1814148610 05.pdf

Rojo, A. y García, D. (2006). La valoración de empresas en España: un estudio empírico. Revista Española de Financiación y Contabilidad, 35(132), 913-934.

Salas de Gómez, N. M. (2018). Desarrollo social de la nueva PDVSA: una mirada desde la perspectiva cualitativa - Primera Parte. Enfoque Disciplinario, 3(1), 18-47. Recuperado a partir de

http://enfoquedisciplinario.org/revista/index.php /enfoque/article/view/11

Sabino, C. (2002). Cómo hacer una tesis. Caracas, Venezuela: Panapo.

Soto Lozada, J. (2016). Factor de competitividad financiera y contable de las organizaciones en Cartagena. Enfoque Disciplinario, 1(1), 1-13. Recuperado a partir de http://enfoquedisciplinario.org/revista/index.php lenfoque/article/view/4

Vergara, J. y De la Espriella, L. (2016). Aplicación de la contabilidad socio-ambiental en las pymes del sector industrial de Cartagena, retos y beneficios. Disponible en: http://revistas.curnvirtual.edu.co/index.php/agla la/article/view/793

Valencia, N. \&otros. (2007). Fundamentos en la transición a los IFRS. Cali: Universidad libre Seccional Cali.

Villarreal, R. (2006). El modelo de competitividad sistémica de los agronegocios en la cadena global de valor. México: IOSD y CECID.

Villarreal, R. (2003). La competitividad sistémica: conceptos y condiciones en México. México: Facultad de Economía, UNAM. 